\title{
Electron-Vector Potential Interaction Hamiltonian
}

\author{
B. Ritchie
}

March 27, 2003

U.S. Department of Energy

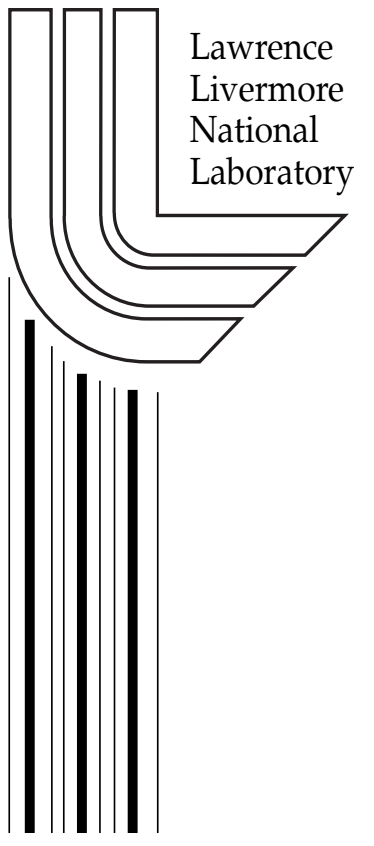




\section{DISCLAIMER}

This document was prepared as an account of work sponsored by an agency of the United States Government. Neither the United States Government nor the University of California nor any of their employees, makes any warranty, express or implied, or assumes any legal liability or responsibility for the accuracy, completeness, or usefulness of any information, apparatus, product, or process disclosed, or represents that its use would not infringe privately owned rights. Reference herein to any specific commercial product, process, or service by trade name, trademark, manufacturer, or otherwise, does not necessarily constitute or imply its endorsement, recommendation, or favoring by the United States Government or the University of California. The views and opinions of authors expressed herein do not necessarily state or reflect those of the United States Government or the University of California, and shall not be used for advertising or product endorsement purposes.

This work was performed under the auspices of the U. S. Department of Energy by the University of California, Lawrence Livermore National Laboratory under Contract No. W-7405-Eng-48.

This report has been reproduced directly from the best available copy.

Available electronically at http://www.doc.gov/bridge

Available for a processing fee to U.S. Department of Energy

And its contractors in paper from

U.S. Department of Energy

Office of Scientific and Technical Information

P.O. Box 62

Oak Ridge, TN 37831-0062

Telephone: (865) 576-8401

Facsimile: (865) 576-5728

E-mail: reports@adonis.osti.gov

Available for the sale to the public from

U.S. Department of Commerce

National Technical Information Service

5285 Port Royal Road

Springfield, VA 22161

Telephone: (800) 553-6847

Facsimile: (703) 605-6900

E-mail: orders@ntis.fedworld.gov

Online ordering: http://www.ntis.gov/ordering.htm

\section{OR}

Lawrence Livermore National Laboratory

Technical Information Department's Digital Library

http://www.llnl.gov/tid/Library.html 
Electron-Vector Potential Interaction Hamiltonian

\author{
Burke Ritchie
}

\author{
University of California \\ Lawrence Livermore National Laboratory \\ Livermore, California 94550
}

We investigate an ambiguity inherent in the definition of the vector potential used in electron-electromagnetic field interactions. Two cases, Zeeman effect and Compton scattering, are studied. 


\section{Introduction}

What is the interaction Hamiltonian $H_{\text {int }}$ for an electron interacting with an electromagnetic vector field? Consider the example of an electron interacting with a spatially uniform magnetic field (Zeeman effect), for which,

$$
\begin{gathered}
\mathrm{H}_{\mathrm{int}}=-\frac{\mathrm{e} \hbar}{2 \mathrm{mc}} \overrightarrow{\mathrm{L}} \cdot \overrightarrow{\mathrm{H}}, \\
\overrightarrow{\mathrm{L}}=-\mathrm{i} \overrightarrow{\mathrm{r}} \times \vec{\nabla},
\end{gathered}
$$

where $\mathrm{H}$ is the magnetic field. Eqs. (1) depend on writing $\mathrm{H}_{\text {int }}$ in terms of the vector potential $\vec{A}$,

$$
\begin{aligned}
\mathrm{H}_{\text {int }} & =\frac{\text { ieћ }}{\mathrm{mc}} \overrightarrow{\mathrm{A}} \cdot \vec{\nabla} \\
\overrightarrow{\mathrm{A}} & =\frac{1}{2} \overrightarrow{\mathrm{H}} \times \overrightarrow{\mathrm{r}}
\end{aligned}
$$

But the vector potential can also be written,

$$
\overrightarrow{\mathrm{A}}=\frac{1}{2} \overrightarrow{\mathrm{H}} \times \overrightarrow{\mathrm{r}}+\vec{\nabla} \mathrm{Q}
$$

since in both cases $\vec{\nabla} \times \vec{A}=\vec{H}$. Q is a scalar field with dimensions of charge. The interaction Hamiltonian now becomes, 


$$
\begin{gathered}
\mathrm{H}_{\text {int }}=\frac{\text { ieћ }}{\mathrm{mc}} \overrightarrow{\mathrm{A}} \cdot \vec{\nabla}+\frac{\text { ieћ }}{2 \mathrm{mc}} \vec{\nabla} \cdot \overrightarrow{\mathrm{A}} \\
\vec{\nabla} \cdot \overrightarrow{\mathrm{A}}=\nabla^{2} \mathrm{Q} .
\end{gathered}
$$

However the two Q-dependent terms in Eq (4a) cancel when they are evaluated to first order using the Schroedinger ground state of an atom $\psi_{0}$. This result is easily seen by parts integration of the matrix element of the first term on the right-hand-side of Eq. (4a),

$$
\left\langle\psi_{0} \vec{A} \cdot \vec{\nabla} \mid \psi_{0}\right\rangle=-\frac{1}{2}\left\langle\psi_{0}|\vec{\nabla} \cdot \vec{A}| \psi_{0}\right\rangle
$$

The two terms however do not cancel in relativistic theory for an electron ignoring spin. In the Klein-Gordon equation,

$$
\left[\frac{1}{c^{2}}\left(i \hbar \frac{\partial}{\partial t}-e \Phi\right)^{2}-\left(-\hbar^{2} \nabla^{2}+\frac{2 i e \hbar}{c} \vec{A} \cdot \vec{\nabla}+\frac{i e h}{c} \vec{\nabla} \cdot \vec{A}+\frac{e^{2}}{c^{2}} A^{2}\right)\right] \psi=m^{2} c^{2} \psi
$$

the $\vec{\nabla} \cdot \overrightarrow{\mathrm{A}}$ contribution is removed by using the Lorentz gauge,

$$
\frac{1}{c} \frac{\partial \Phi}{\partial t}=-\vec{\nabla} \cdot \vec{A}
$$

where $\Phi$ is the scalar potential. This means that the $\vec{A} \cdot \vec{\nabla}$ term in Eq. (6) contributes, in addition to the usual $\overrightarrow{\mathrm{L} \cdot \overrightarrow{\mathrm{H}}}$ term in Eq. (1a), a 
term depending on $Q$, as given by Eqs. (5) and (4b).

What is $Q$ ? It is possible to choose $Q$ to give the Pauli contribution to the Hamiltonian. Let us take,

$$
\mathrm{Q}=\frac{\mathrm{i}}{4 \pi} \int \mathrm{d} \overrightarrow{\mathrm{r}}^{\prime} \frac{\sigma \cdot \mathrm{H}}{\left|\overrightarrow{\mathrm{r}}-\overrightarrow{\mathrm{r}}^{\prime}\right|}
$$

where $\sigma$ is the Pauli vector [1]. Then,

$$
\vec{\nabla} \cdot \vec{A}=\nabla^{2} \mathrm{Q}=-\mathrm{i} \vec{\sigma} \cdot \overrightarrow{\mathrm{H}}
$$

The following point is raised by these considerations. The use of $\vec{A}=\frac{1}{2} \vec{H} \times \vec{r}$ instead of $\vec{A}=\frac{1}{2} \vec{H} \times \vec{r}+\vec{\nabla} Q$ can be viewed as an ambiguity in the definition of the electron-vector field interaction which was removed by Pauli when he postulated the existence of a term going as $\vec{\sigma} \cdot \vec{H}$ to the Hamiltonian. The Dirac equation contributes the Pauli term as well as terms accounting for the magnetic fine structure of the atom. We may ask the question: can all of these spin-dependent contributions be understood from the Klein-Gordon equation once we develop an improved understanding of the quantum vector field $\vec{A}$ and its interaction with a charged 
particle.

We explore this question by studying Compton scattering. It turns out that the propagators for a relativistic free electron and a photon are identical; therefore it is possible to describe Compton scattering by replacing the wavy photon interaction lines and electron propagators of the Feynman graph for Compton scattering in Fig. 1 with electron interaction lines and photon propagators, as shown in Fig. 2. The result, as we show in the next section, is the Klein-Nishina contribution to Compton scattering, whose standard derivation uses the Dirac equation and is diagrammed in Fig. 1.

\section{Compton scattering using photon propagators}

The standard derivation of the Compton cross section uses the Dirac equation, whereupon,

$$
\begin{aligned}
& \frac{\mathrm{d} \sigma}{\mathrm{d} \Omega}=\mathrm{r}_{0}^{2}\left(\frac{\mathrm{k}^{\prime}}{\mathrm{k}}\right)^{2}\left[\left({\hat{\varepsilon} \cdot \varepsilon^{\prime}}^{2}+\frac{\left(\mathrm{k}-\mathrm{k}^{\prime}\right)^{2}}{4 \mathrm{kk^{ \prime }}}\right]\right. \\
& \frac{\mathrm{k}^{\prime}}{\mathrm{k}}=\frac{1}{1+\frac{\hbar \mathrm{k}}{\mathrm{mc}}(1-\cos \theta)}
\end{aligned}
$$

where $r_{0}=e^{2} / m c^{2}$ is the classical-electron radius, $\hat{\varepsilon}, \hat{\varepsilon}$ are unit 
vectors in the incident, scattered directions of polarization of the photon, and $\theta$ is the angle between incident, scattered photon wave vectors $\vec{k}, \vec{k}^{\prime}$. This expression reduces to the classical electromagnetic result [6] when $k^{\prime} / k=1$. The first term on the right side of Eq. (1a) is the sole contribution of Klein-Gordon theory [7]. The second term, first derived by Klein and Nishina [8] using the Dirac equation, is interpreted [9] as the scattering of photons by the permanent magnetic moment of the electron. Both contributions are summarized by the Feynman graph shown in Fig. 1 .

Next we derive the second contribution to Eq. (1a) by using electron interaction lines and the photon propagator, as shown in Fig. 2. We postulate the following Maxwell's equations for the two reaction fields generated by the electron-photon interaction,

$$
\begin{gathered}
\left(\nabla^{2}-\frac{1}{c^{2}} \frac{\partial^{2}}{\partial \mathrm{t}^{2}}\right) \overrightarrow{\mathrm{A}}_{\mathrm{ae}}=4 \pi \mathrm{r}_{0}<\psi_{\mathrm{f} 0}^{*} \overrightarrow{\mathrm{A}}_{0} \psi_{\mathrm{i} 1}+\psi_{\mathrm{i} 1} \overrightarrow{\mathrm{A}}_{0} \Psi_{\mathrm{f} 0}^{*}> \\
\left(\nabla^{2}-\frac{1}{\mathrm{c}^{2}} \frac{\partial^{2}}{\partial \mathrm{t}^{2}}\right) \overrightarrow{\mathrm{A}}_{\mathrm{ea}}=4 \pi \mathrm{r}_{0}<\psi_{\mathrm{f} 1}^{*} \overrightarrow{\mathrm{A}}_{0} \psi_{\mathrm{i} 0}+\psi_{\mathrm{i} 0} \overrightarrow{\mathrm{A}}_{0} \psi_{\mathrm{f} 1^{\prime}}^{*}>
\end{gathered}
$$

where the brackets refer to integration over the space of the number states of the quantized source field, $\overrightarrow{\mathrm{A}}_{0}$, which are unprimed for 
incident and primed for scattered photons. The currents on the right sides of Eqs. (2) are constructed from the Klein-Gordon current [11], generalized to include the number states of $\overrightarrow{\mathrm{A}}_{0}$. There are no further contributions to these currents for an electron initially at rest.

Only the first terms on the right hand sides of Eqs. (2) satisfy energy-momentum conservation. In Eq. (2a) the first term represents annihilation of a photon (with label 1) associated with the initial state $\psi_{i 1}$ (Fig. 2 graph i) of the electron and incident photon. In Eq. (2b) the first term represents creation of a photon from the vacuum state (with label 0 ) associated with the initial state $\psi_{\text {io }}$ (Fig. 2, graph ii) of the electron and scattered photon (cross symmetry graph as in Fig. 1, graph ii).

The source field is defined [4],

$$
\overrightarrow{\mathrm{A}}_{0}=\Sigma \hat{\varepsilon}_{\lambda \sigma}\left[\mathrm{a}_{\lambda \sigma} \mathrm{e}^{\mathrm{i}\left(\overrightarrow{\mathrm{k}}_{\lambda} \overrightarrow{\mathrm{r}}-\omega_{\lambda} \mathrm{t}\right)}+\mathrm{a}_{\lambda \sigma}^{+} \mathrm{e}^{-\mathrm{i}\left(\overrightarrow{\mathrm{k}}_{\lambda} \overrightarrow{\mathrm{r}}-\omega_{\lambda} \mathrm{t}\right)}\right]
$$

$$
\text { where the sum runs over } \lambda \text { modes and } \sigma(\max 2) \text { polarizations, } \hat{\varepsilon}_{\lambda \sigma} \text { are }
$$
unit vectors in the direction of polarization of the photon, and $\mathrm{a}_{\lambda \sigma}$, $\mathrm{a}_{\lambda \sigma}^{+}$are the annihilation, creation operators defined in the usual way 
[4] such that,

$$
\begin{aligned}
& {\left[\mathrm{a}_{\lambda \sigma}, \mathrm{a}_{\lambda^{\prime} \sigma^{\prime}}^{+}\right]=\delta_{\lambda \lambda} \delta_{\sigma \sigma^{\prime}}} \\
& \mathrm{a}_{\lambda \sigma} \mid \mathrm{n}_{\lambda \sigma^{\prime}}>=\sqrt{\mathrm{n}_{\lambda \sigma}} \ln \mathrm{n}_{\lambda \sigma^{-}}-1> \\
& \mathrm{a}_{\lambda \sigma}^{+} \mid \mathrm{n}_{\lambda \sigma^{\prime}}>=\sqrt{\mathrm{n}_{\lambda \sigma}+1} \ln _{\lambda \sigma^{\prime}}+1>.
\end{aligned}
$$

The wave functions on the right side of Eqs. (2) are products of number states of the field for 0 or 1 [Eq.(2a)] or 0 and $1^{\prime}$ [Eq. (2b)] and Klein-Gordon plane-wave states [11], where the one-photon states are defined for a single mode and polarization of the incident [Eq.(2a)] or scattered [Eq. (2b)] photon. For the right side of Eq. (2a),

$$
\begin{aligned}
& \psi_{\mathrm{f} 0}=\sqrt{\frac{m c^{2}}{2 E_{\mathrm{f}} \mathrm{V}}} \mathrm{e}^{\mathrm{i}\left(\overrightarrow{\mathrm{k}_{\mathrm{f}} \mathrm{r}}-\omega_{\mathrm{f}} \mathrm{t}\right)} \mid 0> \\
& \psi_{i 1}=\sqrt{\frac{m c^{2}}{2 E_{i} V}} e^{i\left(\overrightarrow{\hat{k}_{i}} \vec{r}-\omega_{i} t\right)} \mid 1>
\end{aligned}
$$

and similarly for the right side of Eq. (2b) for scattered photons. V 
is the normalization volume and the energies are defined,

$$
\begin{aligned}
& \mathrm{E}_{\mathrm{i}}=\sqrt{\mathrm{m}^{2} \mathrm{c}^{4}+\mathrm{c}^{2} \mathrm{p}_{\mathrm{i}}^{2}}=m c^{2} \\
& \mathrm{E}_{\mathrm{f}}=\sqrt{\mathrm{m}^{2} \mathrm{c}^{4}+\mathrm{c}^{2} \mathrm{p}_{\mathrm{f}}^{2}} .
\end{aligned}
$$

Henceforth 4-vectors are used such that Eq. (5) can be re-written,

$$
\begin{aligned}
& \psi_{\mathrm{f} 0}=\sqrt{\frac{m c^{2}}{2 E_{\mathrm{f}} V}} e^{-\mathrm{i} \kappa_{\mathrm{f}} \mathrm{x}} \mid 0> \\
& \psi_{\mathrm{i} 1}=\sqrt{\frac{m c^{2}}{2 \mathrm{E}_{\mathrm{i}} \mathrm{V}}} \mathrm{e}^{-\mathrm{i} \kappa_{\mathrm{i}} \mathrm{x}} \mid 1>
\end{aligned}
$$

and similarly for the plane-wave states of the electromagnetic field [Eq. (3)]. For example $\kappa x=k_{0} c t-\vec{k} \cdot \vec{r}$, where for photons $k_{0}=k=\omega / c$ for frequency $\omega$, and for electrons $k_{0 j}=E_{j} / h c$. Note the important identities $\kappa^{2}=0$ for photons and $\kappa_{j}^{2}=m^{2} c^{2} / h^{2}$ for electrons. The plane waves are orthonormal in the usual sense [6],

$$
\begin{gathered}
\frac{\hbar V}{m c^{2}(2 \pi)} \int d^{3} k \int d^{3} r \psi_{\kappa}^{*} \overleftrightarrow{\partial}_{0} \Psi_{\kappa^{\prime}}=1 \\
a \vec{\partial}_{0} b=a \frac{\partial b}{\partial t}-b \frac{\partial a}{\partial t} .
\end{gathered}
$$


The solutions to Eqs. (2) are written in momentum space as

functions of $k$ or $k^{\prime}$ and of $t$ in the limit $t \rightarrow \infty$.

$$
\begin{gathered}
\overrightarrow{\mathcal{A}}_{\mathrm{ae}}=-\mathrm{i} \frac{\mathrm{r}_{0}}{\mathrm{~V}} \sqrt{\frac{\mathrm{mc}^{2}}{\mathrm{E}_{\mathrm{f}}}} \frac{\left[(2 \pi) \delta^{4}\left(\kappa_{\mathrm{i}}+\kappa-\kappa_{\mathrm{f}}-\kappa^{\prime}\right)\right]}{2 \mathrm{k}^{\prime}} \hat{\varepsilon} \mathrm{e}^{-\mathrm{ik} \mathrm{k}^{\prime} \mathrm{ct}} \\
\overrightarrow{\mathcal{A}}_{\mathrm{ea}}=\mathrm{i} \frac{\mathrm{r}_{0}}{\mathrm{~V}} \sqrt{\frac{\mathrm{mc}^{2}}{\mathrm{E}_{\mathrm{f}}}} \frac{\left[(2 \pi) \delta^{4}\left(\kappa_{\mathrm{i}}-\kappa^{\prime}-\kappa_{\mathrm{f}}+\kappa\right)\right]}{2 \mathrm{k}} \widehat{\varepsilon^{\prime}} \mathrm{e}^{\mathrm{ikct}}
\end{gathered}
$$

Eniergy-momentum conservation requires that Eqs. (2) be solved using propagators with contours closed in the lower half plane with detours which include (exclude) the positive (negative) frequency root [Eq. (9a)], which is the same as Feynman's boundary condition for the positive-frequency electron propagator [12], and which exclude (include) the positive (negative) frequency root [Eqs. 9b)]. Both waves propagate forward in time, the former for obvious reasons and the latter because the Fourier components of the momentum are reversed relative to the former to satisfy momentum conservation. The different contours insure energy-momentum conservation for the two different currents of Eqs. (2), namely $\kappa_{i}+\kappa=\kappa_{f}+\kappa^{\prime}$ for Eq. (9a) and $\kappa_{i}-\kappa^{\prime}=\kappa_{f}-\kappa$ for Eq. (9b), as graphed in 
Fig. 2, and they are responsible for the negative interference of the Klein-Nishina term [Eq.(1)], as we shall see. The second components of the current in Eqs. (2) do not contribute for the set of boundary conditions just stated because of energy-momentum conditions, $\kappa_{i}=$ $\kappa_{f}+\kappa+\kappa^{\prime}$ for Eq. (9a) and $\kappa_{f}=\kappa_{i}+\kappa+\kappa^{\prime}$ for Eq. (9b), which are impossible to satisfy.

The observable scattering amplitude is written,

$$
\begin{gathered}
\mathrm{S}_{\mathrm{fi}}=\left\langle 1\left|\overrightarrow{\mathcal{A}}_{0}\right| 0\right\rangle \cdot \overrightarrow{\mathcal{A}}_{\mathrm{ae}}+\left\langle 0\left|\overrightarrow{\mathcal{A}}_{0}\right| 1^{\prime}\right\rangle \cdot \overrightarrow{\mathcal{A}}_{\mathrm{ea}} \\
=-\mathrm{i} \frac{\mathrm{r}_{0}}{\mathrm{~V}} \sqrt{\frac{\mathrm{mc}^{2}}{\mathrm{E}_{\mathrm{f}}}} \frac{\left(\mathrm{k}-\mathrm{k}^{\prime}\right)}{2 \mathrm{kk}}\left[(2 \pi) \delta^{4}\left(\kappa_{\mathrm{f}}+\kappa^{\prime}-\kappa_{\mathrm{i}}-\kappa\right)\right] \mathrm{e}^{\mathrm{i}\left(\mathrm{k}-\mathrm{k}^{\prime}\right) \mathrm{ct}} \\
\overrightarrow{\mathcal{A}}_{0}=\hat{\varepsilon} \mathrm{a}^{+} \mathrm{e}^{i k c t}+\hat{\varepsilon}^{\prime} \mathrm{a}^{\prime} \mathrm{e}^{-i \mathrm{k}^{\prime} \mathrm{ct}} .
\end{gathered}
$$

Note the mixing which occurs in the transitions (Fig. 2): $(\kappa, \hat{\varepsilon}) \rightarrow\left(\kappa^{\prime}, \hat{\varepsilon}\right)$ and $\left(\kappa, \hat{\varepsilon^{\prime}}\right) \rightarrow\left(\kappa^{\prime}, \hat{\varepsilon}^{\prime}\right)$ caused by photon propagators which mix $\kappa^{\prime}$ with $\hat{\varepsilon}[\hat{\varepsilon}$ is originally associated with $\kappa$ in Eq. (9a)] and $\kappa$ with $\hat{\varepsilon}^{\prime}\left[\hat{\varepsilon}^{\prime}\right.$ is originally associated with $\kappa^{\prime}$ in Eq. (9b)]. The observable fields are of course the incident and scattered fields, which for the 
quantum theory presented here are the number-state representations of the operator source field $\overrightarrow{\mathrm{A}}_{0}$ [Eq. (3)] and the reaction fields [Eqs. 2)] induced by the currents arising from off-diagonal matrix elements of $\vec{A}_{0}$. The operator fields can be measured diagonally from the number operators $a^{+} a$ (incident field) or $a^{\prime} a^{\prime+}$ (scattered field), or, as in the present application, off-diagonally from the operator product which accumulates after two sucessive operations, namely that of operator a followed by $\mathrm{a}^{+}$[first term on the right side of Eq. (10a)] or that of $a^{\prime+}$ followed by $a^{\prime}$ [second term on the right side of Eq. (10a)]. Thus the first term on the right side of Eq. (10a) represents a photon quantum state measurement of the incident field plus a reaction field at the scattered-photon momentum [graph (i) in Fig. 2]. The second term on the right side of Eq. (10a) represents a photon quantum state measurement of the scattered field plus a reaction field at the incident-field momentum [graph (ii) in Fig. 2]. Other products of the Fourier components of $\overrightarrow{\mathrm{A}}_{0}$ with the momentum-space reaction fields do not form number operators of the incident or scattered fields and are therefore unobservable. 
The cross section differential in the solid angle $d \Omega$ into which the photon is scattered is calculated by taking the squared modulus of Eq. (10a), dividing by $(2 \pi)^{4} \delta^{4}(0)$ and summing over the phase space of the final electron and photon, $\left[\mathrm{V}^{2} /(2 \pi)^{6}\right] d^{3} k_{f} d^{3} k^{\prime}$. Note that the square of $\delta^{4}(\Delta \kappa)$ can be written as the product $\delta^{4}(\Delta \kappa) \delta^{4}(0)$.

On using the identity,

$$
\int d^{3} k^{\prime} \int d^{3} k_{f} \frac{m c^{2}}{E_{f}} \delta^{4}\left(\kappa_{f}+\kappa^{\prime}-\kappa_{i}-\kappa\right)=\int d \Omega \frac{k^{\prime 3}}{k}
$$

which follows from use of the identity for the Lorentz invariant volume element [13],

$$
\int \frac{d^{3} k_{f}}{2 E_{f}}=\int d^{3} k_{f} \int_{-\infty}^{\infty} d\left(\hbar c k_{0 f}\right) \delta\left[\left(\hbar c \kappa_{f}\right)^{2}-m^{2} c^{4}\right] \theta\left(k_{0 f}\right)
$$

and the identity [5],

$$
\int_{0}^{\mathrm{hk}+\mathrm{mc}} \mathrm{d}\left(\hbar \mathrm{k}^{\prime}\right) \delta\left\{2 \mathrm{mc}{ }^{2} \hbar\left[\mathrm{k}-\mathrm{k}^{\prime}-\frac{h \mathrm{kk}}{\mathrm{mc}}(1-\cos \theta)\right]\right\}=\frac{\mathrm{k}^{\prime}}{2 m c^{2} \mathrm{k}}
$$

where use has been made of Eq. (1b), the Klein-Nishina contribution 
immediately follows,

$$
\left(\frac{\mathrm{d} \sigma}{\mathrm{d} \Omega}\right)_{\mathrm{KN}}=\mathrm{r}_{0}^{2}\left(\frac{\mathrm{k}^{\prime}}{\mathrm{k}}\right)^{2} \frac{\left(\mathrm{k}-\mathrm{k}^{\prime}\right)^{2}}{4 \mathrm{kk^{ \prime }}}
$$

When the contribution given by Eq. (14) is added incoherently to the usual Klein-Gordon contribution based on the electron propagator [the first term on the right-hand-side of Eq. (1)], then we obtain the total Compton cross section as given by Eq. (1).

\section{Discussion and Conclusions}

With reference to the modified Feynman graphs in Fig. 2 the scattering amplitude [Eq. (10)] describes the simultaneous processes: (i) annihilation of an incident photon at the incidentelectron interaction line, propagation of the photon at the scattered momentum such that energy-momentum is conserved as shown, creation of the scattered photon at the scattered-electron interaction line; (ii) (cross symmetry graph) creation of the scattered photon at the scattered-electron interaction line, propagation of the photon at the incident momentum such that energy-momentum is conserved as shown, annihilation of the incident photon at the incident-electron interaction line. The 
polarization of the photon does not change because in both processes (i) and (ii) the same photon quantum state exists before and after the reaction. In contrast to conventional Feynman graphs (Fig. 1) for the Diracdescription of Compton scattering, the graphs of Fig. 2 show no electron spin labels but do show photon-state labels. This result suggests an equivalence between photon-free electron spin states and electron-free photon number states.

Acknowledgements. This work was performed under the auspices of the U. S. Department of Energy by Lawrence Livermore National Laboratory under Contract No. W-7405-ENG-48 


\section{References}

1. When we write the vector potential as a complex quantity, as in Eq. (7), then this is tantamount to assuming that the vector potential is a quantum mechanical operator. The quantum mechanical operator nature of the vector potential is well known in the description of spontaneous emission of an excited atom. If the real classical field is used, then unphysical results such as the rate of spontaneous absorption of an atom can be calculated.

2. W. H. Louisell, Quantum Satistical Properties of Radiation (Wiley, New York, 1973), Chapter 5.

3. R. P. Feynman, Quantum Electrodynamics (Benjamin, New York, 1962), pp. 95-104.

4. W. H. Louisell, Quantum Satistical Properties of Radiation (Wiley, New York, 1973), Chapter 4.

5. J. D. Bjorken and S. D. Drell, Relativistic Quantum Mechanics (McGraw Hill, New York, 1964), pp. 127-132. 
6. J. D. Jackson, Classical Electrodynamics, 2nd Ed. (Wiley, New York, 1975), pp. 679-683.

7. J. D. Bjorken and S. D. Drell, Relativistic Quantum Mechanics (McGraw Hill, New York, 1964), pp. 193-195.

8. O. Klein and Y. Nishina, Z. Physik, 52, 853 (1929).

9. J. D. Jackson, Classical Electrodynamics, 2nd Ed. (Wiley, New York, 1975), p. 682.

10. W. H. Louisell, Quantum Satistical Properties of Radiation (Wiley, New York, 1973), pp. 285-296.

11. J. D. Bjorken and S. D. Drell, Relativistic Quantum Mechanics (McGraw Hill, New York, 1964), pp. 186-189.

12. Ibid., pp. 93-95.

13. Ibid, pp. 112-113. 


\section{Figure Captions.}

Figure 1. Standard Feynman graphs for Compton scattering (Ref. 5, Fig. 7 - 10). The wavy lines are photon interaction lines and the solid lines are electron propagator lines.

Figure 2. Feynman graphs with wavy photon interaction lines replaced by electron interaction lines and electron propagators replaced by photon propagators. Graph i: annihilation of an incident photon at the incident-electron interaction line, propagation of the photon at the scattered momentum such that energy-momentum is conserved as shown, creation of the scattered photon at the scattered-electron interaction line; graph ii: (cross symmetry graph) creation of the scattered photon at the scattered-electron interaction line, propagation of the photon at the incident momentum such that energy-momentum is conserved as shown, annihilation of the incident photon at the incident-electron interaction line. 


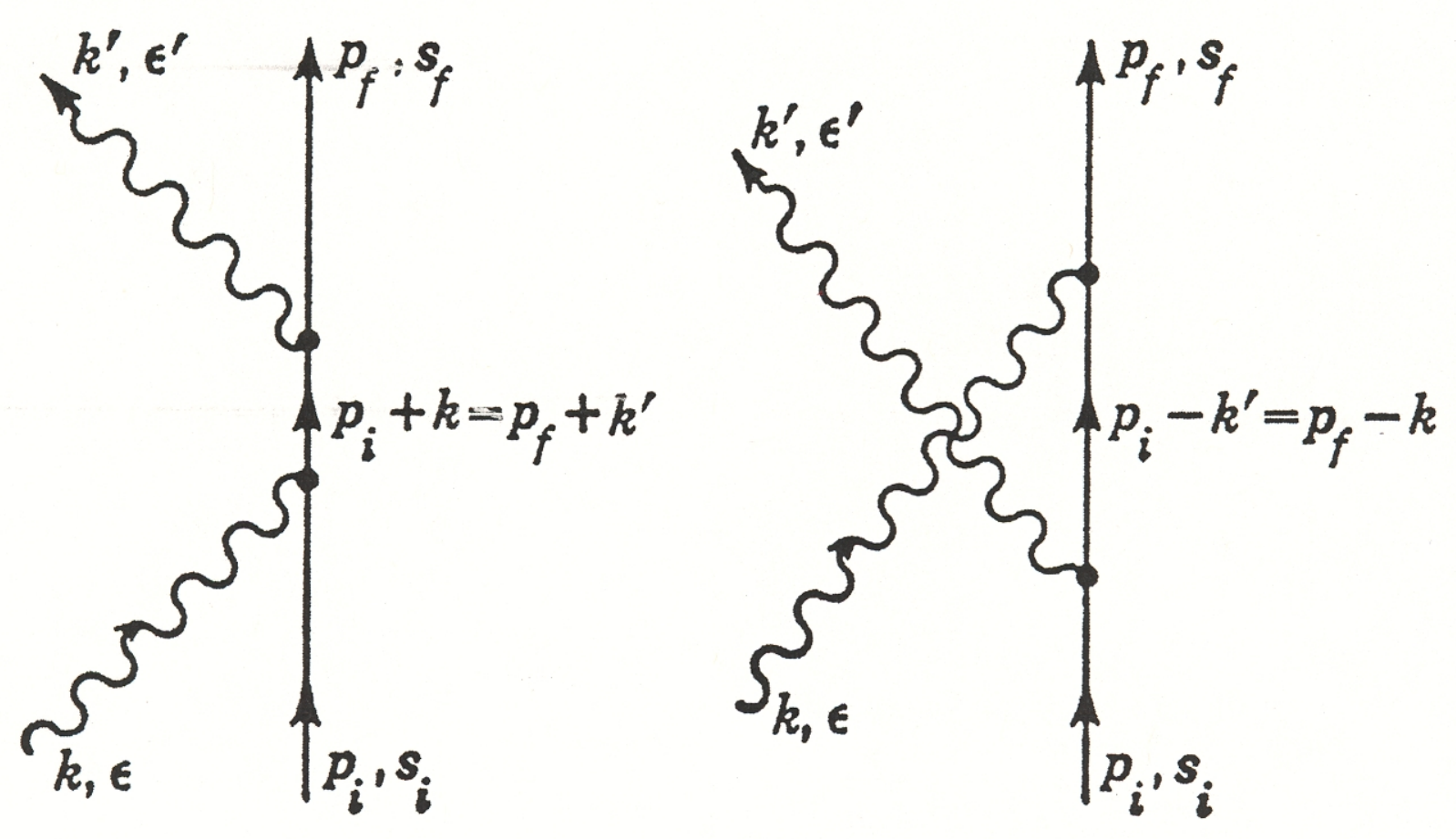

pub_0479913_lad_02 


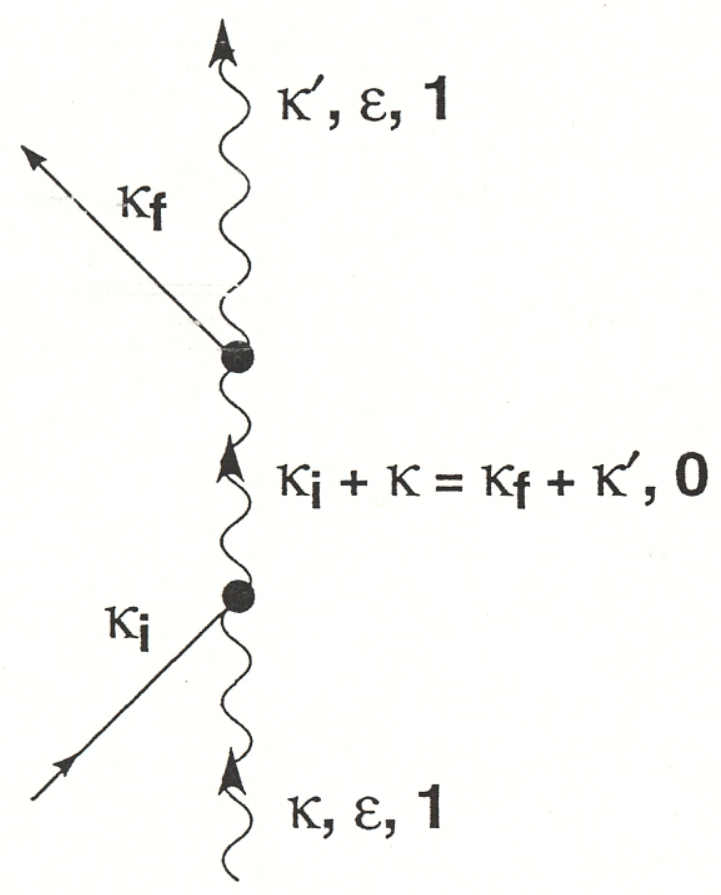

(i)

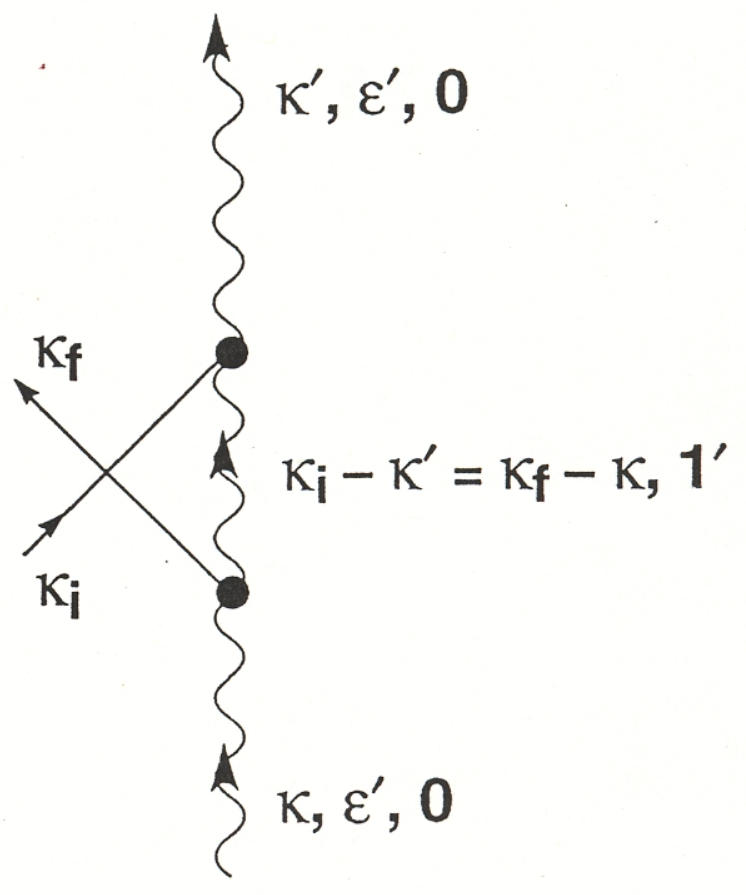

(ii) 